\title{
Ruptured Distal Anterior Inferior Cerebellar Artery Aneurysm Following Stereotactic Irradiation for Vestibular Schwannoma
} -Case Report-

\author{
Shigeru YAMAGUCHI, Tsutomu KATO, Makoto TAKEDA, \\ Hitoshi IKEDA*, and Kei KITAMURA**
}

\begin{abstract}
Departments of Neurosurgery and *Pathology, Hakodate Central Hospital, Hakodate, Hokkaido; ** Department of Radiology, Hakodate Municipal Hospital, Hakodate, Hokkaido
\end{abstract}

\begin{abstract}
A 73-year-old woman presented with diffuse subarachnoid hemorrhage from a ruptured fusiform aneurysm directly arising from the right distal anterior inferior cerebellar artery. Six years before admission, she had undergone stereotactic irradiation for right vestibular schwannoma, under a diagnosis based on neuroimaging. The aneurysm was located within the radiation field. We performed trapping and resection of the aneurysm via a right lateral suboccipital craniotomy, and the patient made a good recovery. Histological examination revealed no evidence of elastic lamina in the aneurysm wall, suggesting pseudoaneurysm caused by radiation-induced vascular injury. Aneurysm formation after radiotherapy is relatively rare and often manifests as fatal hemorrhage. Long-term surviving patients who have received intracranial irradiation should undergo sequential follow up for possible vascular involvement.
\end{abstract}

Key words: anterior inferior cerebellar artery, radiation-induced aneurysm, stereotactic irradiation, subarachnoid hemorrhage, vestibular schwannoma

\section{Introduction}

Radiation-induced intracranial aneurysm is rare, with only about 30 reported cases..$^{1,3,4,6-17)}$ These aneurysms were diagnosed from 10 months $^{7}$ to 21 years ${ }^{4)}$ after radiation therapy. Several patients died of subarachnoid and/or intraventricular hemorrhage secondary to ruptured radiation-induced aneurysms. ${ }^{2,3,15)}$ Autopsy revealed multiple intracranial pseudoaneurysms, one ruptured and others unruptured, probably caused by whole cranial irradiation for malignant brain tumors. ${ }^{2,3,15)}$ Recently, radiationinduced aneurysms have been identified in the field of localized irradiation. . $^{1,6,6,8,11,12,16,17)}$ Almost all these aneurysms arose from the circle of Willis after localized irradiation for suprasellar or parasellar lesions. ${ }^{1,6,8,11,12,17)}$ The mortality associated with ruptured radiation-induced aneurysms is high compared to ruptured congenital saccular aneu- rysms. ${ }^{6,14,17)}$

We present a case of ruptured distal anterior inferior cerebellar artery (AICA) fusiform aneurysm, presumably induced by stereotactic irradiation for vestibular schwannoma, and successfully treated by trapping and resection.

\section{Case Presentation}

A 67-year-old woman underwent stereotactic irradiation (three-dimensional dynamic conformal radiotherapy) of $50 \mathrm{~Gy}$ in 25 fractions for right vestibular schwannoma (Fig. 1), under a diagnosis based on neuroimaging without tissue biopsy. After this treatment, the tumor volume remained stable on magnetic resonance (MR) imaging. Six years later, she was admitted to our hospital because of sudden onset of severe headache associated with nausea and vomiting, followed by disturbance of consciousness.

Received May 15, 2008; Accepted December 26, 2008

Author's present address: Shigeru Yamaguchi, M.D., Department of Neurosurgery, School of Medicine, Hokkaido University, Sapporo, Hokkaido, Japan. 


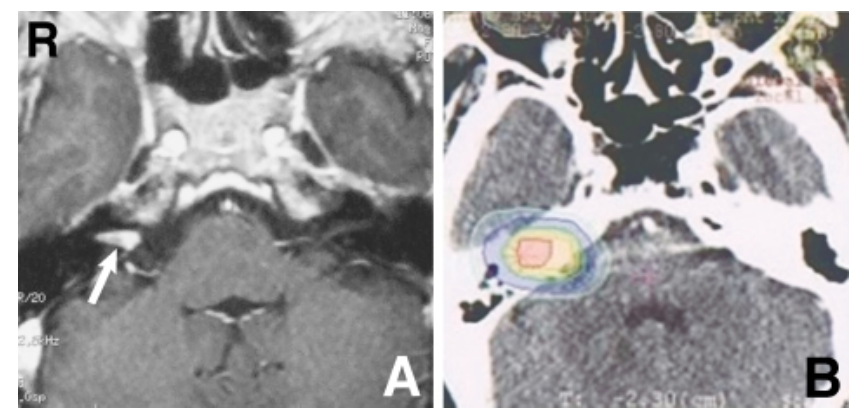

Fig. 1 (A) Axial $\mathrm{T}_{1}$-weighted magnetic resonance image with gadolinium at the initial diagnosis showing a right vestibular schwannoma (arrow). (B) Dose distribution of planning computed tomography scan for stereotactic irradiation (three-dimensional dynamic conformal radiotherapy) for the right vestibular schwannoma. The isodose curves corresponding to $50 \mathrm{~Gy}$ (red), $45 \mathrm{~Gy}$ (yellow), and 25 Gy (blue) are shown.
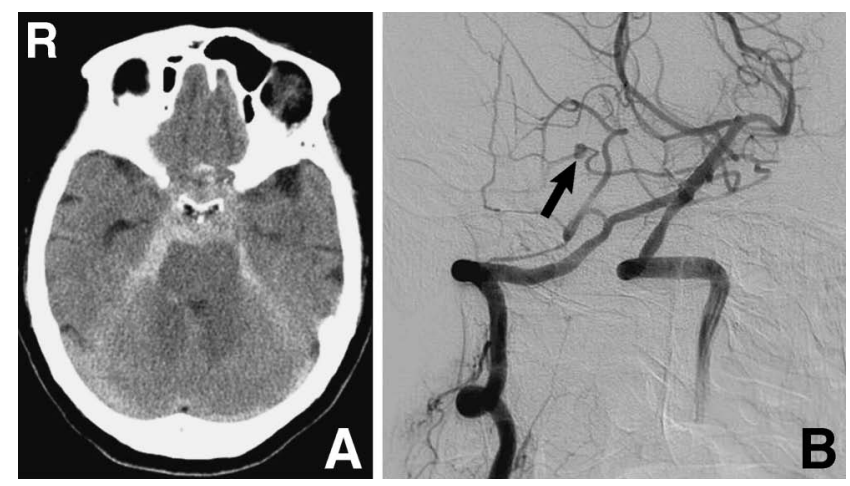

Fig. 2 (A) Computed tomography scan on admission showing diffuse subarachnoid hemorrhage. (B) Right vertebral angiogram revealing a fusiform aneurysm arising directly from a distal site of the right anterior inferior cerebellar artery (arrow), which was located within the radiation field.

Her initial Glasgow Coma Scale score was 14 (E3V5M6), with stable vital signs. Her cranial nerves were intact except for right hearing loss, and motor and sensory examinations revealed no deficits.

Computed tomography (CT) demonstrated diffuse subarachnoid hemorrhage, especially around the cisterns of the posterior fossa, with mild ventricular dilation (Fig. 2A). CT angiography could not confirm the presence of an aneurysm. Right vertebral angiography showed a small aneurysm, approximately $3 \mathrm{~mm}$ in size, arising from the meatal loop of the right AICA (Fig. 2B). This aneurysm was appar-

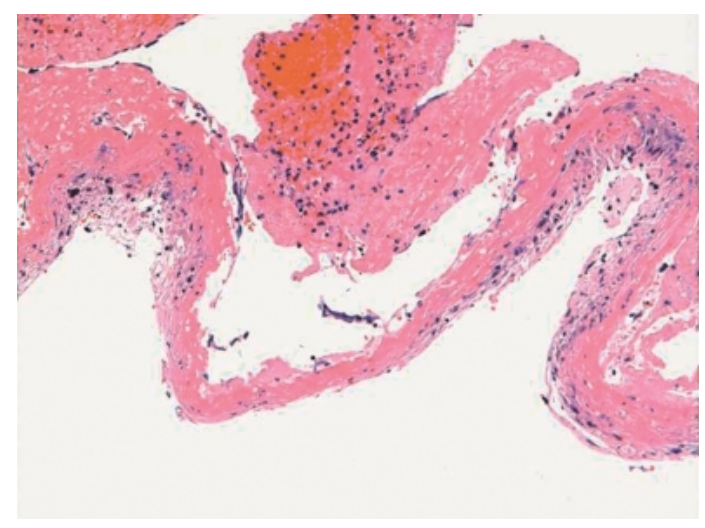

Fig. 3 Photomicrograph showing no residual elastic lamina in the aneurysm wall, indicating a pseudoaneurysm. Hematoxylineosin stain, original magnification $\times 100$.

ently located within the radiation field, and the radiation dose of intracranial portion was probably lower than $50 \mathrm{~Gy}$ according to the dose distribution of planning CT (Fig. 1B).

Surgery via a right lateral suboccipital craniotomy was performed on the day of admission. The aneurysm was not revealed directly because of residual vestibular schwannoma, so partial resection of the tumor was performed to reveal the aneurysm. The aneurysm had a fusiform-like appearance and was not located at the branching point. The rupture site appeared to be near the origin of the parent artery, and this part of the aneurysm wall was unified with the tumor membrane. The aneurysm was trapped using two permanent aneurysm clips, and was resected.

Histological examination identified no evidence of elastic lamina in the aneurysm wall (Fig. 3), which suggested pseudoaneurysm due to radiationinduced vascular injury. The findings confirmed the presumptive diagnosis of schwannoma, and no radiotherapeutic effects were demonstrated in the resected specimens. Postoperative MR imaging revealed a fresh ischemic lesion in the right AICA territory, but no new neurological deficits associated with this infarction. She also underwent ventriculoperitoneal shunting for secondary hydrocephalus. She recovered fully except for moderate right hemifacial palsy and was discharged on the 66 th postoperative day.

\section{Discussion}

The pathogenesis of radiation-induced aneurysms is still speculative, but the mechanism is likely to be similar to that of radiation-induced occlusive vas- 
culopathy, in which initial damage to the endothelium caused by radiation results in aneurysmal changes. ${ }^{14)}$ Autopsy findings of aneurysm walls showed incomplete and fragmented endothelial lining, resulting from focal losses of endothelial cells, and no evidence of elastic lamina. ${ }^{2)}$ Radiation-induced aneurysms differ from congenital aneurysms in location and characteristic histological features according to postmortem examinations of 3 patients with ruptured radiation-induced aneurysms. ${ }^{3)}$ Radiation-induced aneurysms originate directly from the arterial wall, and rupture of the aneurysm wall occurs near its origin and/or at take-off points of arterial branches. In our case, the aneurysm was localized within the radiation field and did not originate at a branching point, and the elastic lamina vanished at the arterial wall. These findings suggest that stereotactic irradiation was responsible for formation of this aneurysm.

The present case provided pathological evidence of a radiation-induced aneurysm arising from a small branch, such as the distal AICA, and was similar to a radiation-induced aneurysm arising from a major trunk..$^{2,3,15)}$ Therefore, formation of an aneurysm after irradiation might occur at any location on vessel included within the radiation fields. In addition, even a small radiation-induced aneurysm is more prone to rupture than other common saccular aneurysms because the pathogenesis may have affected the physical properties of the arterial wall.6)

Sixteen of 26 radiation-induced aneurysms $(61.5 \%)$ ruptured with aneurysmal subarachnoid hemorrhage, and the mortality rate was $42.3 \% .^{14)}$ This high mortality rate was probably due to fatal hemorrhage occurring after diffuse changes in the cerebral vessels. ${ }^{17)}$ Several patients died of fatal subarachnoid hemorrhage before surgical intervention. ${ }^{2,3,10,15)}$ Therefore, early diagnosis is important for radiation-induced aneurysms, although the incidence of aneurysm formation is not high. Careful follow up is necessary in long-term surviving patients to detect both lesions and vascular involvement within the radiation field.

Treatment for radiation-induced intracranial aneurysms includes clip placement, ${ }^{1,4,6-8,17)}$ trapping,9,14) and wrapping the aneurysm and parent artery. ${ }^{4,8,11,12)}$ In addition, parent artery occlusion using an endovascular technique is effective..$^{5,11,16)}$ Radiation-induced aneurysm has a poor prognosis with high risk of mortality if the aneurysm ruptures, so any such suspected aneurysm, even if small, should be treated by surgery or endovascular management. In addition, neck clipping may be impossible because of the formation of pseud- oaneurysm. Postoperative infarction can be avoided if arterial reconstruction is performed, such as a bypass procedure.

The present patient developed a radiation-induced aneurysm despite receiving stereotactic fractionated radiotherapy (three-dimensional dynamic conformal radiotherapy, 50 Gy in 25 fractions), not conventional radiotherapy. Another patient suffered radiationinduced aneurysmal subarachnoid hemorrhage after gamma-knife surgery. ${ }^{16)}$ Therefore, we need to keep in mind that aneurysmal formation can occur after any type of radiotherapy. We would like to recommend that long-term surviving patients who have received intracranial irradiation should undergo sequential follow up for possible vascular involvement. Aggressive surgical intervention or endovascular treatment should be considered for an aneurysm arising within a radiation field, even if the aneurysm is small.

\section{References}

1) Aichholzer M, Gruber A, Haberler C, Bertalanffy A, Slavc I, Czech T: Intracranial hemorrhage from an aneurysm encased in a pilocytic astrocytoma - case report and review of the literature. Childs Nerv Syst 17: 173-178, 2001

2) Azzarelli B, Moore J, Gilmor R, Muller J, Edwards M, Mealey J: Multiple fusiform intracranial aneurysms following curative radiation therapy for suprasellar germinoma. Case report. J Neurosurg 61: 1141-1145, 1984

3) Benson PJ, Sung JH: Cerebral aneurysms following radiotherapy for medulloblastoma. J Neurosurg 70: 545-550, 1989

4) Casey AT, Marsh HT, Uttley D: Intracranial aneurysm formation following radiotherapy. $\mathrm{Br} \mathrm{J}$ Neurosurg 7: 575-579, 1993

5) Cheng KM, Chan CM, Cheung YL, Chiu HM, Tang KW, Law CK: Endovascular treatment of radiationinduced petrous internal carotid artery aneurysm presenting with acute haemorrhage. A report of two cases. Acta Neurochir (Wien) 143: 351-355, 2001

6) Gonzales-Portillo GA, Valdivia JM: Uncommon presentation of pediatric ruptured intracranial aneurysm after radiotherapy for retinoblastoma. Case report. Surg Neurol 65: 391-395, 2006

7) Jensen FK, Wagner A: Intracranial aneurysm following radiation therapy for medulloblastoma. A case report and review of the literature. Acta Radiol 38: 37-42, 1997

8) Maruyama K, Mishima K, Saito N, Fujimaki T, Sasaki $\mathrm{T}$, Kirino $\mathrm{T}$ : Radiation-induced aneurysm and moyamoya vessels presenting with subarachnoid haemorrhage. Acta Neurochir (Wien) 142: 139-143, 2000

9) McConachie NS, Jacobson I: Bilateral aneurysms of 
the cavernous internal carotid arteries following yttrium-90 implantation. Neuroradiology 36: 611-613, 1994

10) Moriyama T, Shigemori M, Hirohata Y, Konishi J, Tokunaga T, Kuramoto S: [Multiple intracranial aneurysms following radiation therapy for pituitary adenoma; a case report]. No Shinkei Geka 20: 487-492, 1992 (Jpn, with Eng abstract)

11) Murakami N, Tsukahara T, Toda H, Kawakami O, Hatano T: Radiation-induced cerebral aneurysm successfully treated with endovascular coil embolization. Acta Neurochir Suppl 82: 55-58, 2002

12) Nishi T, Matsukado Y, Kodama T, Hiraki T: [Multiple intracranial aneurysms following radiation therapy for pituitary adenoma. Case report]. Neurol Med Chir (Tokyo) 27: 224-228, 1987 (Jpn, with Eng abstract)

13) Pereira P, Cerejo A, Cruz J, Vaz R: Intracranial aneurysm and vasculopathy after surgery and radiation therapy for craniopharyngioma: case report. Neurosurgery 50: 885-887, 2002

14) Sciubba DM, Gallia GL, Recinos P, Garonzik IM, Clatterbuck RE: Intracranial aneurysm following radiation therapy during childhood for a brain tumor. Case report and review of the literature. J Neurosurg 105: 134-139, 2006

15) Scodary DJ, Tew JM Jr, Thomas GM, Tomsick T, Liwnicz BH: Radiation-induced cerebral aneurysms. Acta Neurochir (Wien) 102: 141-144, 1990

16) Takao T, Fukuda M, Kawaguchi T, Nishino K, Ito $Y$, Tanaka R, Sato M: Ruptured intracranial aneurysm following gamma knife surgery for acoustic neuroma. Acta Neurochir (Wien) 148: 1317-1318, 2006

17) Yucesoy K, Feiz-Erfan I, Spetzler RF, Han PP, Coons S: Anterior communicating artery aneurysm following radiation therapy for optic glioma: report of a case and review of the literature. Skull Base 14: 169-173, 2004

Address reprint requests to: Shigeru Yamaguchi, M.D., Department of Neurosurgery, School of Medicine, Hokkaido University, North-15, West-7, Kita-ku, Sapporo 060-8638, Japan.

e-mail: yama-shu@med.hokudai.ac.jp 